\title{
BMJ Open Neuroinflammation in cognitive decline post-cardiac surgery (the FOCUS study): an observational study protocol
}

\author{
Annemieke M Peters van Ton (D) , ${ }^{1,2}$ Harmke B. Duindam, ${ }^{1,2}$ Julia van Tuijl (D) ,,3 \\ Wilson WL Li, ${ }^{4}$ Hendrik-Jan Dieker, ${ }^{5}$ Niels P Riksen (1) , ${ }^{2,3}$ FJ Anton Meijer, ${ }^{6,7}$ \\ Roy PC Kessels (D) , ${ }^{8,9}$ Nils Kohn (D) , ${ }^{10}$ Johannes G. van der Hoeven (D) , 1,7 \\ Peter Pickkers (D) , 1,2 Mark Rijpkema (D) ,", Wilson F Abdo (D) 1,2
}

To cite: Peters van Ton AM, Duindam HB, van Tuijl J, et al. Neuroinflammation in cognitive decline postcardiac surgery (the FOCUS study): an observational study protocol. BMJ Open 2021;11:e044062. doi:10.1136/ bmjopen-2020-044062

- Prepublication history and additional supplemental material for this paper are available online. To view these files, please visit the journal online (http://dx.doi.org/10.1136/ bmjopen-2020-044062).

AMPvT and HBD are joint first authors.

Received 22 August 2020 Revised 17 March 2021 Accepted 20 April 2021

Check for updates

(C) Author(s) (or their employer(s)) 2021. Re-use permitted under CC BY-NC. No commercial re-use. See rights and permissions. Published by BMJ.

For numbered affiliations see end of article.

Correspondence to

Dr Wilson F Abdo;

f.abdo@radboudumc.nl

\section{ABSTRACT}

Introduction Postoperative cognitive dysfunction occurs frequently after coronary artery bypass grafting (CABG). The underlying mechanisms remain poorly understood, but neuroinflammation might play a pivotal role. We hypothesise that systemic inflammation induced by the surgical trauma could activate the innate immune (glial) cells of the brain. This could lead to an exaggerated neuroinflammatory cascade, resulting in neuronal dysfunction and loss of neuronal cells. Therefore, the aims of this study are to assess neuroinflammation in vivo presurgery and postsurgery in patients undergoing major cardiac surgery and investigate whether there is a relationship of neuroinflammation to cognitive outcomes, changes to brain structure and function, and systemic inflammation.

Methods and analysis The FOCUS study is a prospective, single-centre observational study, including 30 patients undergoing elective on-pump CABG. Translocator protein (TSP0) positron emission tomography neuroimaging will be performed preoperatively and postoperatively using the second generation tracer ${ }^{18} \mathrm{~F}-\mathrm{DPA}-714$ to assess the neuroinflammatory response. In addition, a comprehensive cerebral MRI will be performed presurgery and postsurgery, in order to discover newly developed brain and vascular wall lesions. Up to 6 months postoperatively, serial extensive neurocognitive assessments will be performed and blood will be obtained to quantify systemic inflammatory responses and peripheral immune cell activation.

Ethics and dissemination Patients do not benefit directly from engaging in the study, but imaging neuroinflammation is considered safe and no side effects are expected. The study protocol obtained ethical approval by the Medical Research Ethics Committee region ArnhemNijmegen. This work will be published in peer-reviewed international medical journals and presented at medical conferences.

Trial registration number NCT04520802.

\section{INTRODUCTION}

Background and rationale

Coronary artery bypass grafting (CABG) has been associated with postoperative cerebral complications. ${ }^{1-4}$ These may occur directly
Strengths and limitations of this study

- This study is the first to examine in vivo neuroinflammation using translocator protein positron emission tomography neuroimaging both prior to and after cardiac surgery.

- This study combines state of the art neuroimaging with longitudinal neuropsychological examinations and detailed immunological analyses.

- Extensive neuropsychological examinations at four different time points enable us to detect even subtle changes in cognitive function.

- This study is powered to detect the association between neuroinflammation and cognitive decline between baseline and hospital discharge, when the incidence of cognitive dysfunction is highest.

- The power of this pilot study is inadequate to adjust for confounders.

postsurgery, like stroke or delirium, but can also have long-term sequelae such as postoperative cognitive dysfunction (POCD) and dementia. The incidence of POCD ranges from $20 \%-70 \%$ in the first week after surgery to $10 \%-40 \%$ in the following months but may increase again from one postoperative year onwards. ${ }^{15}$ Furthermore, patients have a 1.7-fold increased risk to develop new incident Alzheimer's disease within 6years after undergoing a CABG, compared with patients undergoing a percutaneous coronary intervention. ${ }^{6}$

Development of POCD is presumably related to perioperative brain hypoperfusion, cerebral microembolisation, haemodilution, hypercoagulability, cerebral hyperthermia and inadequate glucose homoeostasis. $^{2}$ In addition, systemic inflammation is hypothesised as an important (and possibly treatable) factor for POCD pathogenesis. ${ }^{7-9}$ Cardiac surgery produces substantial systemic inflammation 
(reflected by leucocytosis and significant dysregulation of cytokines and other inflammatory mediators, affecting various physical processes) due to multiple stimuli such as sternotomy, extracorporeal circulation (ECC), associated transient endotoxaemia and aortic cross-clamping. Systemic inflammation can result in increased communication and signalling from the periphery to the brain. ${ }^{1011}$ As a result, systemic inflammation may induce activation of the innate immune cells of the brain, the microglia and astrocytes, leading to a neuroinflammatory response. ${ }^{12}$ Animal models demonstrated that systemic administration of low-dose endotoxin even leads to long-term inflammatory reprogramming of microglia. ${ }^{13}$ Interestingly, research has shown that neuroinflammation is associated with cognitive dysfunction and neurodegenerative disease. ${ }^{14} 15$ Given these associations, we hypothesise that occurrence of POCD in patients undergoing $\mathrm{CABG}$ is mediated through the occurrence of neuroinflammation.

Positron emission tomography (PET) enables the imaging of glial activation in the central nervous system (CNS) using radiolabelled antagonists of translocator protein (TSPO). ${ }^{16}{ }^{17}$ TSPO is mainly expressed as a transmembrane protein on mitochondria of microglia and astrocytes in the CNS. ${ }^{18}$ As TSPO expression is upregulated during neuroinflammatory processes, it makes a promising biomarker for imaging neuroinflammation. ${ }^{19}$ Up to now, TSPO neuroimaging has been applied in a wide variety of neurodegenerative and psychiatric conditions, showing associations between neuroinflammation and cognitive decline. ${ }^{20-22}$ Recently, first in vivo evidence of microglial activity in response to systemic inflammation has been shown in patients and healthy volunteers. ${ }^{23-26}$ However, human in vivo data on neuroinflammation in patients undergoing major cardiothoracic surgery are still lacking.

This study, therefore, aims to assess neuroinflammation presurgery and postsurgery in vivo in patients undergoing elective cardiac surgery. Given the presumed association between neuroinflammation and cognitive decline, quantifying the extent of neuroinflammation postsurgery and its relation to POCD will provide us with important insights for future (interventional) research.

\section{Objectives}

Our primary objective is to assess whether neuroinflammation is more pronounced in patients with cognitive decline at hospital discharge after CABG surgery, compared to patients without cognitive decline.

Secondary objectives:

1. To study the relation between the neuroinflammatory response and structural or functional changes to the brain postoperatively.

2. To study the relation between neuroinflammation and cognitive decline at 6 weeks and 6 months post-cardiac surgery.
3. To study the relation between neuroinflammation and postoperative delirium.

4. To study the relation between the perioperative systemic immune responses and neuroinflammation.

\section{METHODS AND ANALYSIS}

\section{Study design}

The FOCUS study (neuroinflammation in cognitive decline post-çardiac surgery) is a single-centre observational time-series design study investigating neuroinflammation, systemic inflammation and neuropsychological performance before-and-after CABG.

\section{Study population and recruitment}

All patients planned for elective CABG in an academic hospital in the Netherlands are screened. Table 1 presents the inclusion and exclusion criteria. The age criterion of 50 years or older is chosen for two reasons. First, older adults are more prone to subsequent long-term cognitive decline after cardiac surgery. ${ }^{27}{ }^{28}$ Second, ageing is associated with a more exaggerated neuroinflammatory response following systemic inflammation. ${ }^{29}$

Screening and enrolment logs will be maintained for all patients. After written informed consent is obtained, a blood sample will be genotyped for rs6971 polymorphism using Taqman analysis on a 7500 Fast Real-Time PCR System (ThermoFisher Scientific, Waltham, USA). Low-affinity TSPO binding patients for the radiotracer used in PET imaging will be excluded from participation. In our predominantly Caucasian cohort, the estimated percentage of low affinity binders is below $10 \% .^{30}$

\section{Data collection}

Patients' demographics and information regarding their surgery indication, treatments, pre-existing comorbidity (Charlson Comorbidity Index), ${ }^{31} 32$ (re)admission, disease severity and mortality risk, length of mechanical ventilation, length of stay at the intensive care unit (ICU) and occurrence of delirium will be retrieved from the patients' medical files. Table 2 shows an overview of events for this study, which will be defined subsequently in more detail.

\section{Cerebral imaging}

Dynamic brain PET/CT scans will be obtained preoperatively and on the fourth (range: 3rd-7th) postoperative day. Dynamic imaging of the head will be performed for $60 \mathrm{~min}$ after intravenous injection of the radiolabelled TSPO antagonist ${ }^{18}$ F-DPA-714 (18-fluoride labeled N,N-diethyl-2-[4-(3-fluoroethoxy) phenyl]-5,7dimethylpyrazolo [1,5-a]pyrimidine-3-acetamide), a second generation TSPO ligand. This generation of TSPO ligands outperforms the first generation TSPO tracer, isoquinoline carboxamide $\left({ }^{11} \mathrm{C}-\mathrm{PK} 11195\right)$, on TSPO binding affinity and PET imaging properties, but requires polymorphism genotyping and stratifying according to binding affinity status. ${ }^{33} \mathrm{~A}$ dose of $\sim 200 \mathrm{MBq}$ of ${ }^{18} \mathrm{~F}$-DPA714 will be administered as a slow bolus injection during 
Table 1 Inclusion and exclusion criteria

Inclusion criteria

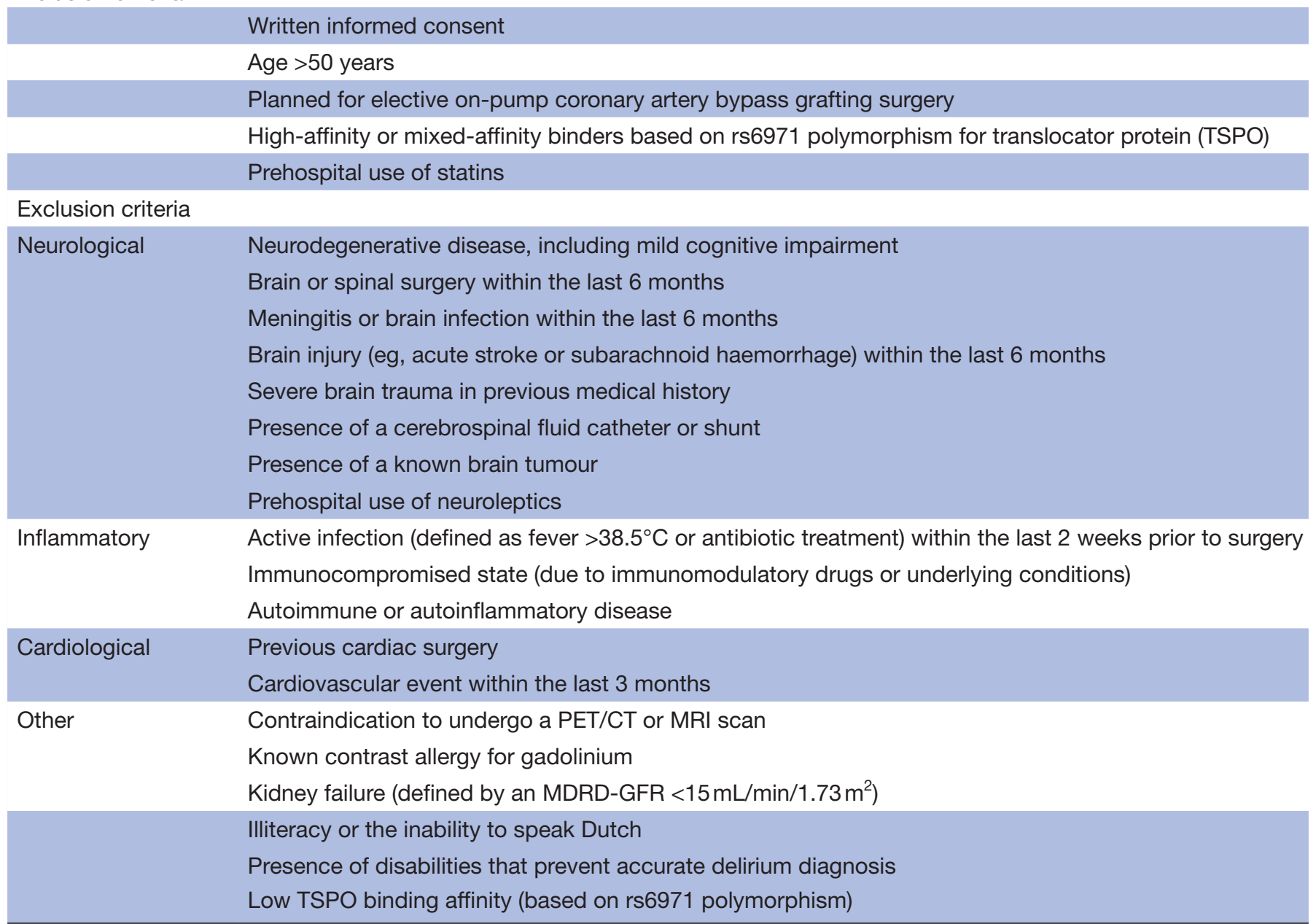

CT, computed tomography; MDRD-GFR, Modification of Diet in Renal Disease - Glomerular Filtration Rate; MRI, magnetic resonance imaging; PET, positron emission tomography; TSPO, translocator protein.

40 s. Scans are obtained on a Siemens Biograph mCT hybrid PET/CT scanner (Siemens, Erlangen, Germany). A low-dose CT will be acquired for attenuation correction and anatomical reference. For pharmacokinetic analysis of the PET data arterial blood sampling will be used.

In addition, a 3 Tesla brain MRI (Siemens TIM TRIO, Erlangen, Germany) will be performed on the same days as the PET/CT scans. T1-weighted MR images will be obtained to co-register with the PET images for anatomical reference. Whole brain grey matter, as well as regions of interest (ROI), will be delineated using probabilistic brain region templates. The slice thickness will be $1 \mathrm{~mm}$. Additionally, the following MRI sequences will be performed: T2-weighed, susceptibility weighted imaging (SWI), fluid-attenuated inversion recovery (FLAIR), diffusion tensor imaging (DTI), resting state functional MRI (fMRI), time of flight (TOF) MR angiogram of the circle of Willis and precontrast and postcontrast three-dimensional high-resolution T1-weighted SPACE sequence to visualise vessel wall abnormalities. In order to visualise cerebral vessel wall abnormalities, $0.1 \mathrm{mmol} /$ $\mathrm{kg}$ gadobutrol (Gadovist) contrast agent will be administered intravenously. Details on MRI settings are provided in online supplemental appendix 1.

A senior neuroradiologist blinded to all other data will systematically quantify newly developed lesions postsurgery. This enables us to analyse the relationship between neuroinflammation, neuropsychological decline and cerebral lesion load. To evaluate changes in brain functional connectivity due to an acute immune response, a resting-state fMRI measurement is implemented comparing resting state connectivity in stress-related brain circuits presurgery and postsurgery. ${ }^{34}$ A previous study found positive associations between alterations in resting-state functional connectivity in the brain's default mode network and global cognitive change after cardiac surgery, ${ }^{36}$ and the current study enables us to extend these findings by studying the relationship with (neuro)inflammation.

\section{Blood sampling}

Blood samples will be obtained at baseline preoperatively (T0, concomitant with the PET-scan), intraoperatively at 


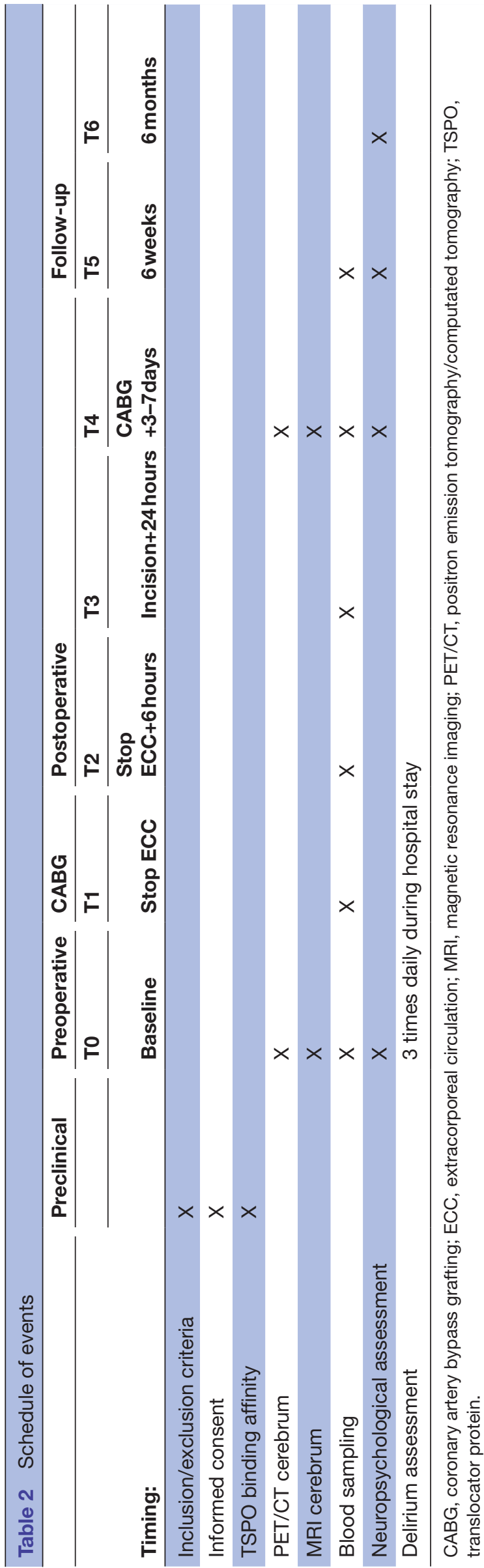

the stop of ECC (T1), 6 hours after stop ECC (T2) and 24 hours after incision (T3). The timing of blood draws is based on the peak of the systemic inflammatory response post-cardiac surgery, as shown in previous studies we performed in patients undergoing cardiac surgery. ${ }^{37} 38$ Additionally, blood samples will be collected concomitant with the second PET/CT-scan (T4) and 6 weeks postoperatively (T5) to explore to what extent the inflammatory response persists in time.

At all time points, common blood parameters will be measured, including haemoglobin, leucocyte and thrombocyte count, and circulating pro-inflammatory and anti-inflammatory cytokines (including tumour necrosis factor alpha (TNF- $\alpha$ ), interleukin (IL)-6, IL-1 $\beta$, IL-10, and IL-1 receptor antagonist (IL-1RA)) by using simultaneous Luminex assays. Blood will be centrifuged at $2000 \mathrm{~g}$ for $10 \mathrm{~min}$ and the plasma will be stored at $-80^{\circ} \mathrm{C}$ for possible future additional testing in line with the objective of this protocol. In addition, danger-associated molecular patterns (including high mobility group box 1, heat shock protein-70, calgranulin-C (S100A12), calprotectin (S100A8/9), nuclear DNA and mitochondrial DNA) will be measured as reported previously, ${ }^{39}$ in plasma centrifuged for a second time at high speed $(16000 \mathrm{~g})$.

Additionally, ex vivo production of cytokines by stimulated isolated peripheral blood mononuclear cells will be measured, including TNF- $\alpha$, IL-6, IL-1 $\beta$, monocyte chemoattractant protein-1 and IL-10. Flow cytometry analysis of whole blood will be performed as described previously, ${ }^{40}$ to study the inflammatory phenotype of the cells (including expression of human leucocyte antigen-DR, C-C chemokine receptor type 2, CD11b, CD14 and $\mathrm{CD} 16$ ).

Arterial blood samples will be taken immediately before the ${ }^{18}$ F-DPA-714 injection and during the PET-scan to measure the time course of radioactivity in plasma. In addition to this pharmacokinetic sampling, blood samples will be used to assess the ratio of ${ }^{18} \mathrm{~F}-\mathrm{DPA}-714$ and its metabolites in order to correct the arterial input function for metabolite formation.

\section{Neuropsychological assessment}

A trained psychologist will perform neuropsychological assessments preoperatively (T0), at hospital discharge (T4), after 6 weeks (T5) and 6 months (T6) follow-up. These assessments are in line with the recommendation for neuropsychological research in cardiac surgery patients. ${ }^{42}$ Table 3 lists the full test battery used for the neuropsychological assessments as well as the self-report questionnaires. Tests were selected based on sensitivity to detect even subtle deterioration in cognitive performance, with a focus on the domains executive functioning, memory, speed of processing and language. We will use parallel versions of these tests to account for materialspecific practice effects after repeated assessment. ${ }^{43}$

During hospitalisation, screening of delirium is standard of care. Confusion Assessment Method for the $\mathrm{ICU}^{44}$ or Delirium Observation Screening scores ${ }^{45} 46$ at 
Table 3 Summary of neuropsychological testing

\section{Neuropsychological assessments}

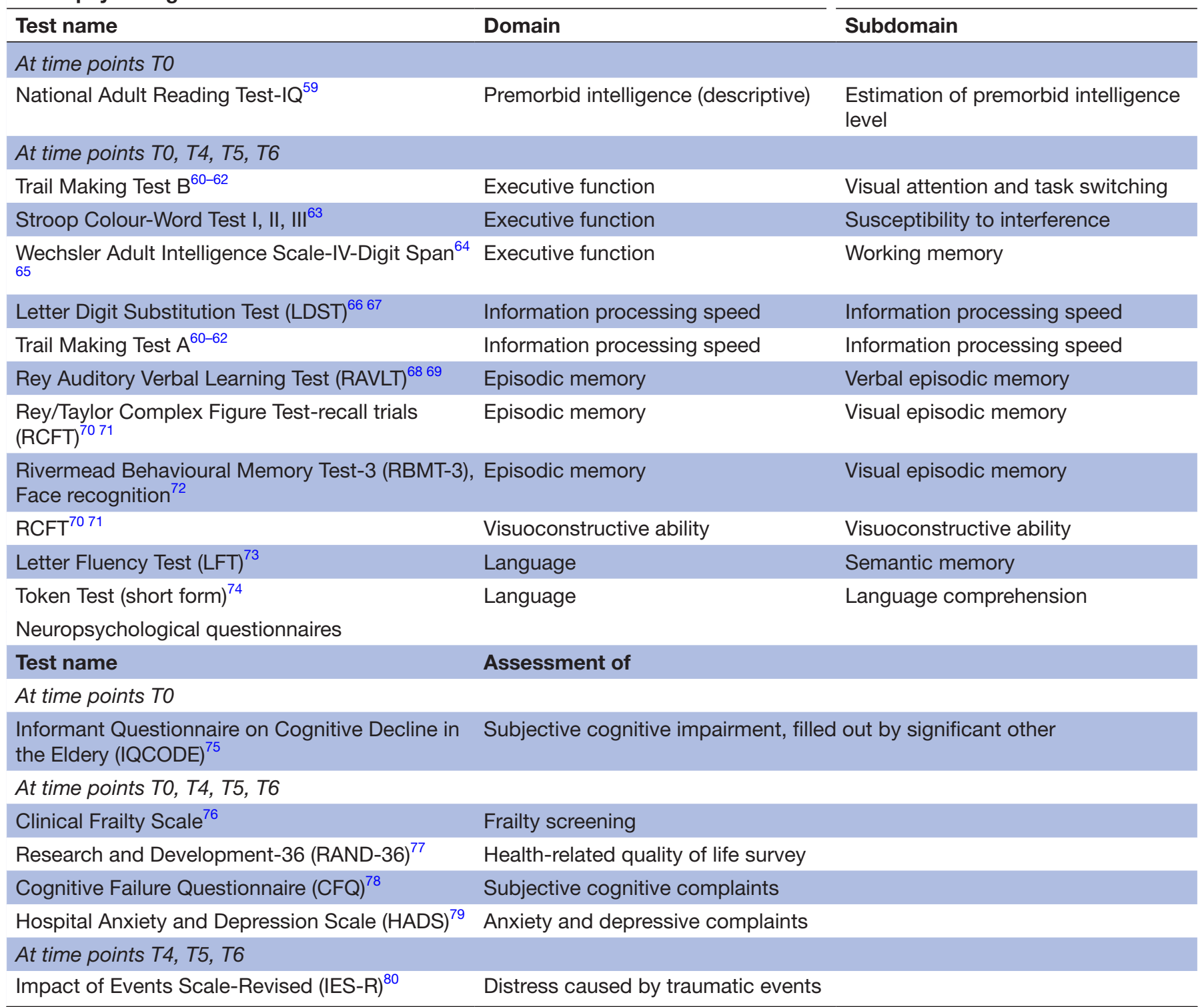

the cardiothoracic ward will be performed three times a day. A dedicated senior delirium researcher or neurologist will validate the diagnosis of delirium, using DSM-5 (Diagnostic and Statistical Manual of Mental Disorders 5 th edition) criteria for delirium. ${ }^{47}$

\section{Cardiac surgery}

Perioperative care will be delivered according to the regular clinical protocol for CABG. This minimises the risk of potential confounders due to variability in medical management.

\section{Data analysis}

Pharmacokinetic modelling of dynamic PET data will be performed in PMOD software (PMOD Technologies, Zürich, Switzerland). Binding potential $\left(\mathrm{BP}_{\mathrm{ND}}\right)$ and volume of distribution $\left(\mathrm{V}_{\mathrm{T}}\right)$ in several regions of interest (ROIs) will be determined using the 2-tissue compartmental model for each scan. Predefined ROIs include the frontal, temporal, parietal and occipital lobes, amygdala, hippocampus, thalamus, cerebellum and the brain stem.

For each patient, ${ }^{18} \mathrm{~F}$-DPA-714 $\mathrm{BP}_{\mathrm{ND}}$ will be measured in the ROI for baseline and post-surgery PET scans. Since $\mathrm{BP}_{\mathrm{ND}}$ is proportional to the availability of TSPO binding sites in the brain, an increase in $\mathrm{BP}_{\mathrm{ND}}$ reflects an increase in glial activation. In addition, we will measure $\mathrm{V}_{\mathrm{T}}$ which recently has been shown to mainly reflect changes in peripheral tracer binding during systemic inflammation, rather than changes in TSPO expression in the brain. ${ }^{25}$ (and unpublished data from our group) Therefore, both outcome measurements are required to validate these recent findings and assess glial activation accurately. Study investigators who analyse the cerebral imaging data will be blinded for inflammatory mediator results and cognitive outcomes. 
No consensus about the definition of POCD has been achieved in the literature thus far. ${ }^{5849}$ At all time points, neuropsychological tests of our patients will be compared with available normative data, adjusted for age and educational level, resulting in standardised z-scores. Overall domain z-scores will be calculated by averaging the z-scores of the individual tests within a specific domain. Calculation of test performance will be supervised by an experienced clinical neuropsychologist. Additionally, z-scores of each individual test will be clinically classified as either being within the normal range (0), below average (1) or impaired (2), compared with the aforementioned normative data. 'Normal performance' is defined as performance above $-1 \mathrm{SD}$ from the normative mean. 'Below average' as between $-1 \mathrm{SD}$ and $-1.65 \mathrm{SD}$ from the normative mean (the lowest $16 \%$ of the normal population) and 'impaired' as below -1.65 SD from the normative mean (the lowest $5 \%$ of the normal population).$^{50} 51$ Thus, an overall weighted performance score will be calculated for all five cognitive domains. A cognitive domain will be clinically classified as impaired when the average weighted score of all tests within the domain is $>1$. We will define the presence of POCD when 1) patients are impaired in one or more domains at hospital discharge, compared with baseline, or 2) when the overall weighted performance score deteriorated (from classification score 0 or 1 to 1 or 2 , respectively) in more than one domain at hospital discharge compared with baseline. Accordingly, all patients will be dichotomised into two groups: with or without POCD at hospital discharge.

The percentage change in TSPO expression (postoperative vs preoperative) will be calculated and reported as mean $\pm \mathrm{SE}$ of the mean (SEM) if normally distributed. This value can be compared using unpaired t-tests between both groups with Bonferroni post hoc correction, and additionally, linear mixed models will be performed with the presence of POCD and brain region modelled as fixed effects, and subject ID as random effect. Additionally, the (change in) mean of the cognitive domain scores is analysed as continuous dependent outcome, with (change in) TSPO expression and (change in) systemic inflammatory markers as predictors in multiple linear regression models. Age, sex, Clinical Frailty Scale, Charlson Comorbidity Index, Hospital Anxiety and Depression Scale and the RAND-36 item health survey at inclusion can be included as covariates. Multiple logistic regression analysis is performed to correct for possible confounders such as newly developed structural brain lesions on MRI.

The trajectory of systemic inflammatory parameters and differences in time points will be measured with repeated measures one-way analysis of variance or linear mixed models in case of missings. In addition, correlations between systemic inflammatory markers and TSPO expression will be studied using Pearson for parametric or Spearman for non-parametric data. Unpaired t-tests will be applied to compare TSPO expression between patients with and without a delirious episode during hospital admission.
All MRI images will be evaluated without prior notice of any clinical parameter. White matter lesions are defined as hyperintense lesions on FLAIR MRI without corresponding cerebrospinal fluid like hypo-intense lesions on the T1-weighted image. Lacunar infarcts are defined as hypointense areas $>2 \mathrm{~mm}$ and $\leq 15 \mathrm{~mm}$ on FLAIR and T1. ${ }^{52}$ Territorial infarcts are defined as hyperintense lesions on FLAIR, and hypointense lesions on T1 image. ${ }^{52}$ And finally, microbleeds are defined as small, homogenous, round foci of low signal intensity on $\mathrm{T} 2 *$ weighted images of $<10 \mathrm{~mm}$ in diameter. ${ }^{53}$

Analyses of functional and structural brain images will be performed using FSL (FMRIB's Software Library, Oxford, UK).$^{54}$ After preprocessing and denoising, subject-wise spatial maps of with-in network brain connectivity of the salience, executive control and default mode networks will be compared with non-parametric tests for mean differences. Vascular wall enhancement will be compared between the preoperative and postoperative assessments with paired t-tests or the Wilcoxon signed rank test if the data are non-parametric.

Descriptive statistics will be performed using IBM-SPSS software, version 25 . Mean $( \pm$ SEM) or median $(\mathrm{IQR})$ will be presented depending on the distribution of the data. Alpha will be set at 0.05 throughout.

After the first five patients we will schedule a technical interim analysis to establish whether a tissue reference model is a reliable, non-invasive method for pharmacokinetic analysis of TSPO neuroimaging during systemic inflammation-induced neuroinflammation. If this or other (mathematical) methods can be reliably validated in our cohort, there will be no longer need for arterial blood sampling.

\section{Sample size calculation}

No data on the degree of glial activation after systemic inflammatory responses in post-cardiac surgery patients are available. Research on cognitive dysfunction after cardiac surgery estimates a prevalence of approximately $50 \%$ at hospital discharge. ${ }^{1548}$ Therefore, we will define two groups based on the presence or absence of cognitive dysfunction at hospital discharge.

Previous studies in dementia patients observed a 15\%-35\% higher PET tracer uptake in patients with cognitive impairment compared with healthy controls, with an SD of $30 \% .^{55} 56$ Therefore, we assume that patients with cognitive dysfunction at hospital discharge after cardiac surgery will have a $30 \%$ higher delta tracer uptake compared with patients without cognitive decline. To assess a $30 \%$ higher delta tracer uptake in patients with cognitive dysfunction, an unpaired two-sample t-test results in 13 patients per group with a power of $80 \%$ and a one-sided alpha of 0.05 . In order to account for lower increments we will include 15 patients per group resulting in a power of $85 \%$ with an alpha of 0.05 to differentiate an increase of $30 \%$ in delta tracer uptake. Consequently, we will include a total of 30 patients, assuming that $50 \%$ will have cognitive decline at hospital discharge. The 
investigator can decide to withdraw a subject from the study for (1) urgent medical reasons or (2) if a protocol violation occurs or (3) if the subject is lost to follow-up. Replacement of individuals will not be necessary in this observational cohort once both PET scans are performed (ie, primary objective has been met).

Sample size calculations were conducted for the primary objective solely, considering cognitive decline at hospital discharge. Assessment of long-term cognitive outcomes (up to 6 months) together with assessment of our secondary outcomes has been set up in an exploratory setting. The data of this study will be important to calculate the power and feasibility of a subsequent prospective project focused on long-term outcomes.

\section{Interim analysis with futility stop}

An interim analysis will be performed after the first 15 subjects to validate that CABG surgery indeed induces a neuroinflammatory response, which will be assessed by TSPO PET imaging. An independent data safety and monitoring board (DSMB), composed by two clinical experts in the field of nuclear medicine and neurology, and a biostatistician, will analyse whether increased PET tracer uptake is observed after CABG surgery compared with the preoperative baseline tracer uptake. If no trend towards a significant difference $(p>0.10)$ will be observed, the inclusion will be stopped to prevent futility. Furthermore an adaptive power analysis will be performed by the unblinded statistician DSMB member during the interim analysis to determine whether the study is underpowered to fulfil the primary objective, and whether the sample size should be adapted. The pooled SD of the delta PET tracer uptake will be calculated from the first 15 patients of which approximately $50 \%$ have cognitive disorders at hospital discharge. Using this pooled SD the sample size calculation will be performed again. If the ratio between this new sample size and the original is greater than 1 , the sample size will be adapted if deemed feasible. The investigators will remain blinded for cognitive outcomes until the end of the study.

\section{Study period}

The study started enrolling patients in March 2019. The estimated study enrolment completion date is anticipated in the beginning of 2022. Please note that this manuscript was finalised prior to the interim analyses.

\section{Ethics and dissemination}

The study is conducted according to the principles of the Declaration of Helsinki and in accordance with the Dutch Medical Research Involving Human Subjects Act (WMO) and Good Clinical Practice guidelines. The study obtained ethical approval by the Medical Research Ethics Committee (MREC) region Arnhem-Nijmegen (CMO 2016-2598). The study is registered in the ClinicalTrials. gov database (NCT04520802). The burden of the study protocol consists of two PET/CT-scans and two cerebral MRIs, additional blood sampling before, during and after surgery and four neuropsychological examinations, two during hospital admission and two follow-up visits during the first 6 months after hospital discharge.

\section{Patient and public involvement statement}

The hypothesis of this study was conceived with the help of patients through outpatient clinical follow-up after an ICU admission. Through patient experience of different long-term consequences, this study's endpoints involves cognitive performance, psychological symptoms and quality of life. A patient member of the MREC judged the study protocol for feasibility, burden and understandability of patient information.

\section{Data management}

Data will be handled confidentially and pseudonymously. Study data will be deidentified and a secured subject identification code list will be kept and stored separately from the data. This observational study uses an electronic remote data capture system. All missing and ambiguous data will be queried. The investigator will permit studyrelated monitoring, audits and regulatory inspection at their site, providing access to source data/documents. In all cases, it remains the responsibility of the investigator to ensure that data are accurate. Coded data will be kept after closure of the study and can only be used for ancillary studies after strict approval of the principal investigator. Anonymised data can be shared with other organisations for academic research, consensus development or other projects aimed at advancement of knowledge in this area. Body materials consisting of blood will be preserved in a coded form for 10 years for possible follow-up studies. The MREC will be consulted before body material is used for follow-up research.

\section{Public disclosure and publication policy}

The results of this study will be published, regardless of whether these are positive, negative or inconclusive, in peer-reviewed international (open access) medical journals and presented at medical conferences. In addition, a summary of the results of this study will be published on the website of the funding agency The Netherlands Organisation for Health Research and Development (ZonMw).

\section{Relevance of findings}

Cerebral dysfunction after cardiac surgery occurs frequently and may severely affect patients' daily lives. Due to the lack of research data within this area, the pathophysiology of cerebral dysfunction postsurgery is unknown. Therefore, there are currently no interventions available to prevent or treat this deterioration. The FOCUS study will quantify glial activation, which is suggested to be important in this pathophysiology, and relate this to cognition, structural and functional changes to the brain and systemic inflammation. This adds to previous and ongoing observational work perioperatively combining blood and cerebrospinal fluid parameters with MR neuroimaging, cognition and electroencephalogram 
recordings. ${ }^{5758}$ Our study combines state of the art molecular and MR neuroimaging techniques, elaborates longitudinal neuropsychological examinations and comprehensive immunological laboratory tests. Objective neurocognitive examinations will be performed at four different time points, up to 6 months postoperatively, enabling us to detect even subtle changes in cognition. Better understanding of the pathogenesis of POCD could direct neuroscientists towards the development of targets for future interventions. This imaging paradigm could provide an approach to examine the efficacy of such interventions in clinical studies.

In addition, the knowledge obtained with this study is of importance for patients and healthcare professionals as well. The participating patient will not directly benefit from study participation. However, they will be followed with regard to possible complaints in light of potential post-cardiac surgery cerebral dysfunction. This will increase the awareness of the participating patients and caregivers with respect to such complaints. This might benefit the patient and caregivers as it will decrease uncertainty about the nature of the complaints when they occur. Furthermore, this study searches for a biological explanation for post-surgery cerebral complaints which are often not understood or classified as functional.

Several limitations need to be addressed. First, as this study is powered at cognitive decline at hospital discharge, it is not powered to study the association between glial activation and cognitive decline after 6 months. The second limitation concerns the arterial sampling during the dynamic PET-scans. Automated arterial sampling, which is frequently used in other studies using outpatient clinic patients, leads to a significant loss of blood per scan. Loss of such an amount of blood in cardiac surgery patients is not preferable as it could result in a significant health risk for these critically ill patients. Therefore we will sample manually, which reduces blood volume loss. Manual sampling results in less sampling points and could therefore lead to a slightly less accurate plasma activity curve. Unfortunately, TSPO expression is not specific to microglia and astrocytes, and the measured PET signal can be affected by recruitment of peripheral monocytes to the brain, adherence of circulating leucocytes to the vascular epithelium, or TSPO expression in neurons or vascular endothelial cells. Finally, the power of this pilot study is inadequate to allow adjustment for all potential confounding factors.

Imaging neuroinflammation as proposed is safe, as corroborated by existing human and animal data. In addition, imaging neuroinflammation could lead to potential prognostic and interventional targets that could revolutionise healthcare for this large group of patients.

\section{Author affiliations}

${ }^{1}$ Department of Intensive Care Medicine, Radboud university medical center, Nijmegen, The Netherlands

${ }^{2}$ Radboud Institute for Molecular Life Sciences, Radboud university medical center, Nijmegen, The Netherlands
${ }^{3}$ Department of Internal Medicine, Radboud university medical center, Nijmegen, The Netherlands

${ }^{4}$ Department of Cardiothoracic Surgery, Radboud university medical center, Nijmegen, The Netherlands

${ }^{5}$ Department of Cardiology, Radboud university medical center, Nijmegen, The Netherlands

${ }^{6}$ Department of Medical Imaging, Radboud university medical center, Nijmegen, The Netherlands

${ }^{7}$ Radboud Institute for Health Sciences, Radboud university medical center, Nijmegen, The Netherlands

${ }^{8}$ Department of Medical Psychology, Radboud university medical center, Nijmegen, The Netherlands

${ }^{9}$ Donders Center for Cognition, Radboud University, Nijmegen, The Netherlands ${ }^{10}$ Donders Institute for Brain, Cognition and Behaviour, Cognitive Neuroscience, Radboud university medical center, Nijmegen, The Netherlands

Twitter Peter Pickkers @ICresearch_Rumc and Wilson F Abdo @WilsonF_Abdo

Acknowledgements The authors are very grateful for the help of our colleagues at the department of Radiology and Nuclear Medicine, especially Gerben Franssen, Desirée Bos, Peter Laverman, Jurrian Butter, Peter Kok, Maichel van Riel, Michel de Groot, Marijke Hogenkamp and Judith Thijssen. In addition, we wish to thank Petra Budde, Jacqueline Blaauwbroek and Tanja Derks from the cardiothoracic planning office, for their help and flexibility in scheduling study patients. Assistance provided by the psychologists Chiara Fasotti, Claudette van Roij, Maud van Dorst and Lizzy van der Horst in performing the neuropsychological examinations was greatly appreciated. Furthermore we wish to thank Jelle Gerretsen and Matthijs Kox from the Intensive Care Research Unit for their input in the immunological assays in this study. Finally, this study could not be conducted without the help of the cardiothoracic surgeons, anaesthesiologists, intensivists and ICU nurses.

Contributors WFA conceived the study idea. AMPvT and WFA developed the study design to which JvT, WWL, H-JD, NPR, FJAM, RPCK, NK, JGvdH, PP and MR contributed. AMPVT and HBD drafted the manuscript. All authors were involved in the editing of the manuscript and read and approved the final manuscript.

Funding WFA and this study were supported by a research grant from the Netherlands Organisation for Health Research and Development (ZonMW Clinical Fellowship grant 90715610).

Disclaimer This funding agency had no role in the concept, design or writing of this study.

Competing interests None declared.

Patient consent for publication Not required.

Provenance and peer review Not commissioned; externally peer reviewed.

Supplemental material This content has been supplied by the author(s). It has not been vetted by BMJ Publishing Group Limited (BMJ) and may not have been peer-reviewed. Any opinions or recommendations discussed are solely those of the author(s) and are not endorsed by BMJ. BMJ disclaims all liability and responsibility arising from any reliance placed on the content. Where the content includes any translated material, BMJ does not warrant the accuracy and reliability of the translations (including but not limited to local regulations, clinical guidelines, terminology, drug names and drug dosages), and is not responsible for any error and/or omissions arising from translation and adaptation or otherwise.

Open access This is an open access article distributed in accordance with the Creative Commons Attribution Non Commercial (CC BY-NC 4.0) license, which permits others to distribute, remix, adapt, build upon this work non-commercially, and license their derivative works on different terms, provided the original work is properly cited, appropriate credit is given, any changes made indicated, and the use is non-commercial. See: http://creativecommons.org/licenses/by-nc/4.0/.

\section{ORCID iDs}

Annemieke M Peters van Ton http://orcid.org/0000-0001-7979-5149

Julia van Tuijl http://orcid.org/0000-0001-8042-6121

Niels P Riksen http://orcid.org/0000-0001-9197-8124

Roy PC Kessels http://orcid.org/0000-0001-9500-9793

Nils Kohn http://orcid.org/0000-0002-1954-2753

Johannes G. van der Hoeven http://orcid.org/0000-0001-5362-0244

Peter Pickkers http://orcid.org/0000-0002-1104-4303

Mark Rijpkema http://orcid.org/0000-0002-8495-4703

Wilson F Abdo http://orcid.org/0000-0002-5783-8643 


\section{REFERENCES}

1 Greaves D, Psaltis PJ, Ross TJ, et al. Cognitive outcomes following coronary artery bypass grafting: a systematic review and metaanalysis of 91,829 patients. Int J Cardiol 2019;289:43-9.

2 Berger M, Terrando N, Smith SK, et al. Neurocognitive function after cardiac surgery: from phenotypes to mechanisms. Anesthesiology 2018;129:829-51.

3 Brown $\mathrm{CH}$, Probert J, Healy R, et al. Cognitive decline after delirium in patients undergoing cardiac surgery. Anesthesiology 2018;129:406-16.

4 Sauër AC, Veldhuijzen DS, Ottens TH, et al. Association between delirium and cognitive change after cardiac surgery. $\mathrm{Br} J$ Anaesth 2017;119:308-15.

5 Bruce K, Smith JA, Yelland G, et al. The impact of cardiac surgery on cognition. Stress and Health 2008;24:249-66.

6 Lee TA, Wolozin B, Weiss KB, et al. Assessment of the emergence of Alzheimer's disease following coronary artery bypass graft surgery or percutaneous transluminal coronary angioplasty. J Alzheimers Dis 2005;7:319-24.

7 Wan Y, Xu J, Ma D, et al. Postoperative impairment of cognitive function in rats: a possible role for cytokine-mediated inflammation in the hippocampus. Anesthesiology 2007;106:436-43.

8 Cibelli M, Fidalgo AR, Terrando N, et al. Role of interleukin-1beta in postoperative cognitive dysfunction. Ann Neurol 2010;68:360-8.

9 Cao X-Z, Ma H, Wang J-K, et al. Postoperative cognitive deficits and neuroinflammation in the hippocampus triggered by surgical trauma are exacerbated in aged rats. Prog Neuropsychopharmacol Biol Psychiatry 2010;34:1426-32.

10 Varatharaj A, Galea I. The blood-brain barrier in systemic inflammation. Brain Behav Immun 2017;60:1-12.

11 D'Mello C, Swain MG. Immune-to-Brain communication pathways in inflammation-associated sickness and depression. Curr Top Behav Neurosci 2017;31:73-94.

12 Perry $\mathrm{VH}$, Holmes $\mathrm{C}$. Microglial priming in neurodegenerative disease. Nat Rev Neurol 2014;10:217-24

13 Wendeln A-C, Degenhardt K, Kaurani L, et al. Innate immune memory in the brain shapes neurological disease hallmarks. Nature 2018;556:332-8.

14 Dheen ST, Kaur C, Ling E-A. Microglial activation and its implications in the brain diseases. Curr Med Chem 2007;14:1189-97.

15 Streit WJ, Mrak RE, Griffin WST. Microglia and neuroinflammation: a pathological perspective. J Neuroinflammation 2004;1:14.

16 Turkheimer FE, Rizzo G, Bloomfield PS, et al. The methodology of TSPO imaging with positron emission tomography. Biochem Soc Trans 2015;43:586-92.

17 Song YS. Perspectives in TSPO PET imaging for neurologic diseases. Nucl Med Mol Imaging 2019;53:382-5.

18 Casellas P, Galiegue S, Basile AS. Peripheral benzodiazepine receptors and mitochondrial function. Neurochem Int 2002;40:475-86.

19 Lee Y, Park Y, Nam H, et al. Translocator protein (TSPO): the new story of the old protein in neuroinflammation. BMB Rep 2020;53:20-7.

20 Bradburn S, Murgatroyd C, Ray N. Neuroinflammation in mild cognitive impairment and Alzheimer's disease: a meta-analysis. Ageing Res Rev 2019;50:1-8.

21 Plavén-Sigray P, Matheson GJ, Collste K, et al. Positron emission tomography studies of the glial cell marker translocator protein in patients with psychosis: a meta-analysis using individual participant data. Biol Psychiatry 2018;84:433-42.

22 Enache D, Pariante CM, Mondelli V. Markers of central inflammation in major depressive disorder: a systematic review and metaanalysis of studies examining cerebrospinal fluid, positron emission tomography and post-mortem brain tissue. Brain Behav Immun 2019;81:24-40.

23 Sandiego CM, Gallezot J-D, Pittman B, et al. Imaging robust microglial activation after lipopolysaccharide administration in humans with PET. Proc Natl Acad Sci U S A 2015;112:12468-73.

24 Forsberg A, Cervenka S, Jonsson Fagerlund M, et al. The immune response of the human brain to abdominal surgery. Ann Neurol 2017;81:572-82.

25 Nettis MA, Veronese M, Nikkheslat N, et al. PET imaging shows no changes in TSPO brain density after IFN- $\alpha$ immune challenge in healthy human volunteers. Trans/ Psychiatry 2020;10:89.

26 Peters van Ton AM, Leijte GP, Franssen GM, et al. Human in vivo neuroimaging to detect reprogramming of the cerebral immune response following repeated systemic inflammation. Brain Behav Immun 2021. doi:10.1016/j.bbi.2021.04.004. [Epub ahead of print: 09 Apr 2021].
27 Newman MF, Kirchner JL, Phillips-Bute B, et al. Longitudinal assessment of neurocognitive function after coronary-artery bypass surgery. N Engl J Med 2001;344:395-402.

28 Glumac S, Kardum G, Karanovic N. Postoperative cognitive decline after cardiac surgery: a narrative review of current knowledge in 2019. Med Sci Monit 2019;25:3262-70.

29 Norden DM, Godbout JP. Review: microglia of the aged brain: primed to be activated and resistant to regulation. Neuropathol Appl Neurobiol 2013;39:19-34.

30 Owen DR, Yeo AJ, Gunn RN, et al. An 18-kDa translocator protein (TSPO) polymorphism explains differences in binding affinity of the PET radioligand PBR28. J Cereb Blood Flow Metab 2012;32:1-5.

31 Charlson ME, Pompei P, Ales KL, et al. A new method of classifying prognostic comorbidity in longitudinal studies: development and validation. J Chronic Dis 1987;40:373-83.

32 Quan H, Li B, Couris CM, et al. Updating and validating the Charlson comorbidity index and score for risk adjustment in hospital discharge Abstracts using data from 6 countries. Am J Epidemiol 2011;173:676-82.

33 Owen DRJ, Gunn RN, Rabiner EA, et al. Mixed-affinity binding in humans with 18-kDa translocator protein ligands. J Nucl Med 2011;52:24-32.

34 Hermans EJ, Henckens MJAG, Joëls M, et al. Dynamic adaptation of large-scale brain networks in response to acute stressors. Trends Neurosci 2014;37:304-14.

35 Kohn N, Hermans EJ, Fernández G. Cognitive benefit and cost of acute stress is differentially modulated by individual brain state. Soc Cogn Affect Neurosci 2017;12:1179-87.

36 Browndyke JN, Berger M, Harshbarger TB, et al. Resting-State functional connectivity and cognition after major cardiac surgery in older adults without preoperative cognitive impairment: preliminary findings. J Am Geriatr Soc 2017;65:e6-12.

37 Hoedemaekers C, van Deuren M, Sprong T, et al. The complement system is activated in a biphasic pattern after coronary artery bypass grafting. Ann Thorac Surg 2010;89:710-6.

38 Hoedemaekers CW, Pickkers P, Netea MG, et al. Intensive insulin therapy does not alter the inflammatory response in patients undergoing coronary artery bypass grafting: a randomized controlled trial [ISRCTN95608630]. Crit Care 2005;9:R790-7.

39 Leijte GP, Custers H, Gerretsen J, et al. Increased plasma levels of danger-associated molecular patterns are associated with immune suppression and postoperative infections in patients undergoing cytoreductive surgery and hyperthermic intraperitoneal chemotherapy. Front Immunol 2018;9:663.

40 Noz MP, Hartman YAW, Hopman MTE, et al. Sixteen-Week physical activity intervention in subjects with increased cardiometabolic risk shifts innate immune function towards a less proinflammatory state. J Am Heart Assoc 2019;8:e013764.

41 Leijte GP, Rimmelé T, Kox M, et al. Monocytic HLA-DR expression kinetics in septic shock patients with different pathogens, sites of infection and adverse outcomes. Crit Care 2020;24:110.

42 Murkin JM, Newman SP, Stump DA, et al. Statement of consensus on assessment of neurobehavioral outcomes after cardiac surgery. Ann Thorac Surg 1995;59:1289-95.

43 Beglinger LJ, Gaydos B, Tangphao-Daniels O, et al. Practice effects and the use of alternate forms in serial neuropsychological testing. Arch Clin Neuropsychol 2005;20:517-29.

44 Ely EW, Margolin R, Francis J, et al. Evaluation of delirium in critically ill patients: validation of the confusion assessment method for the intensive care unit (CAM-ICU). Crit Care Med 2001;29:1370-9.

45 Schuurmans M, Donders AR, Shortridge-Baggett LM, et al. Delirium case finding: pilot testing of a new screening scale for nurses. J Am Geriatr Soc 2002;50:S3.

46 Schuurmans MJ, Shortridge-Baggett LM, Duursma SA. The delirium observation screening scale: a screening instrument for delirium. Res Theory Nurs Pract 2003;17:31-50.

47 American Psychiatric Association,. Diagnostic and statistical manual of mental disorders. 5th Edition. Arlington, VA: American Psychiatric, 2013.

48 Rudolph JL, Schreiber KA, Culley DJ, et al. Measurement of postoperative cognitive dysfunction after cardiac surgery: a systematic review. Acta Anaesthesiol Scand 2010;54:663-77.

49 Needham MJ, Webb CE, Bryden DC. Postoperative cognitive dysfunction and dementia: what we need to know and do. $\mathrm{Br} J$ Anaesth 2017;119:1115-25.

50 van den Berg E, Kessels RPC, de Haan EHF, et al. Mild impairments in cognition in patients with type 2 diabetes mellitus: the use of the concepts $\mathrm{MCl}$ and CIND. J Neurol Neurosurg Psychiatry 2005;76:1466-7

51 Reukers DFM, Aaronson J, van Loenhout JAF, et al. Objective cognitive performance and subjective complaints in patients 
with chronic $Q$ fever or $Q$ fever fatigue syndrome. BMC Infect Dis 2020;20:397.

52 Hervé D, Mangin J-F, Molko N, et al. Shape and volume of lacunar infarcts: a 3D MRI study in cerebral autosomal dominant arteriopathy with subcortical infarcts and leukoencephalopathy. Stroke 2005;36:2384-8.

53 Ashburner J, Friston KJ. Unified segmentation. Neuroimage 2005;26:839-51.

54 Jenkinson M, Beckmann CF, Behrens TEJ, et al. FSL. Neuroimage 2012;62:782-90.

55 Suridjan I, Pollock BG, Verhoeff NPLG, et al. In-vivo imaging of grey and white matter neuroinflammation in Alzheimer's disease: a positron emission tomography study with a novel radioligand, [18F]FEPPA. Mol Psychiatry 2015;20:1579-87.

56 Varrone A, Oikonen V, Forsberg A, et al. Positron emission tomography imaging of the $18-\mathrm{kDa}$ translocator protein (TSPO) with [18F]FEMPA in Alzheimer's disease patients and control subjects. Eur J Nucl Med Mol Imaging 2015;42:438-46.

57 Berger M, Oyeyemi D, Olurinde MO, et al. The INTUIT study: investigating neuroinflammation underlying postoperative cognitive dysfunction. J Am Geriatr Soc 2019;67:794-8.

58 Danielson M, Wiklund A, Granath F, et al. Neuroinflammatory markers associate with cognitive decline after major surgery: findings of an explorative study. Ann Neurol 2020;87:370-82.

59 Nelson HE, O'Connell A. Dementia: the estimation of premorbid intelligence levels using the new adult reading test. Cortex 1978;14:234-44.

60 Partington JE, Leiter RG. Partington's pathways test. Psychological Service Center Journal 1949;1:11-20.

61 Reitan RM, Wolfson D. The halstead-reitan neuropsychological test battery. Tuscon, AZ: Neuropsychological Press, 1985

62 Larrabee GJ, Millis SR, Meyers JE. Sensitivity to brain dysfunction of the Halstead-Reitan vs an ability-focused neuropsychological battery. Clin Neuropsychol 2008;22:813-25.

63 Stroop JR. Studies of interference in serial verbal reactions. J Exp Psychol 1935:18:643-62.

64 Kessels RPC, Molleman PW, Oosterman JM. Assessment of working-memory deficits in patients with mild cognitive impairment and Alzheimer's dementia using Wechsler's working memory index. Aging Clin Exp Res 2011;23:487-90.
65 Wechsler D. Wechsler adult intelligence Scale-Fourth editionNederlandse bewerking. Technische handleiding en Afname en scorehandleiding. Amsterdam: Pearson Assessment and Information BV, 2012.

66 Natu MV, Agarwal AK. Digit letter substitution test (DLST) as an alternative to digit symbol substitution test (DSST). Hum Psychopharmacol 1995;10:339-43.

67 Jolles J, Houx PJ, van Boxtel MPJ. e.a., the Maastricht aging study: determinants of cognitive aging. Maastricht: Neuropsych Publishers, 1995.

68 Schmidt M. Rey auditory verbal learning test: Ravlt: a Handbook. Los Angeles: Western Psychological Services, 1996.

69 Saan RJ, Deelman BG. De 15-woordentest A en B. Afdeling Neuropsychologie, AZG: Groningen, 1986.

70 Rey A. Psychological examination of traumatic encephalopathy. Clinical Neuropsychologist 1993;7:3-21.

71 Meyers JE, Meyers KR. Rey complex figure test and recognition trial: professional manual. Odessa: Psychological Assessment Resources, 1995.

72 Wilson B, Cockburn J, Baddeley A. The Rivermead behavioural memory test manual. Bury St Edmunds: Thames Valley Test Co, 1985.

73 Mulder JLD, P.H.; Dekker R. Word-fluency test/figure fluency test (wft/fft). PITS Uitgeverij BV: Leiden, 2006.

74 De Renzi E, Faglioni P. Normative data and screening power of a shortened version of the Token test. Cortex 1978;14:41-9.

75 Jorm AF, Jacomb PA. The informant questionnaire on cognitive decline in the elderly (IQCODE): socio-demographic correlates, reliability, validity and some norms. Psychol Med 1989;19:1015-22.

76 Rockwood K, Song X, MacKnight C, et al. A global clinical measure of fitness and frailty in elderly people. CMAJ 2005;173:489-95.

77 Hays RD, Sherbourne CD, Mazel RM. The Rand 36-Item health survey 1.0. Health Econ 1993;2:217-27.

78 Broadbent DE, Cooper PF, FitzGerald P, et al. The cognitive failures questionnaire (CFQ) and its correlates. Br J Clin Psychol 1982;21:1-16.

79 Spinhoven P, Ormel J, Sloekers PP, et al. A validation study of the hospital anxiety and depression scale (HADS) in different groups of Dutch subjects. Psychol Med 1997;27:363-70.

80 Creamer M, Bell R, Failla S. Psychometric properties of the Impact of Event Scale - Revised. Behav Res Ther 2003;41:1489-96. 